
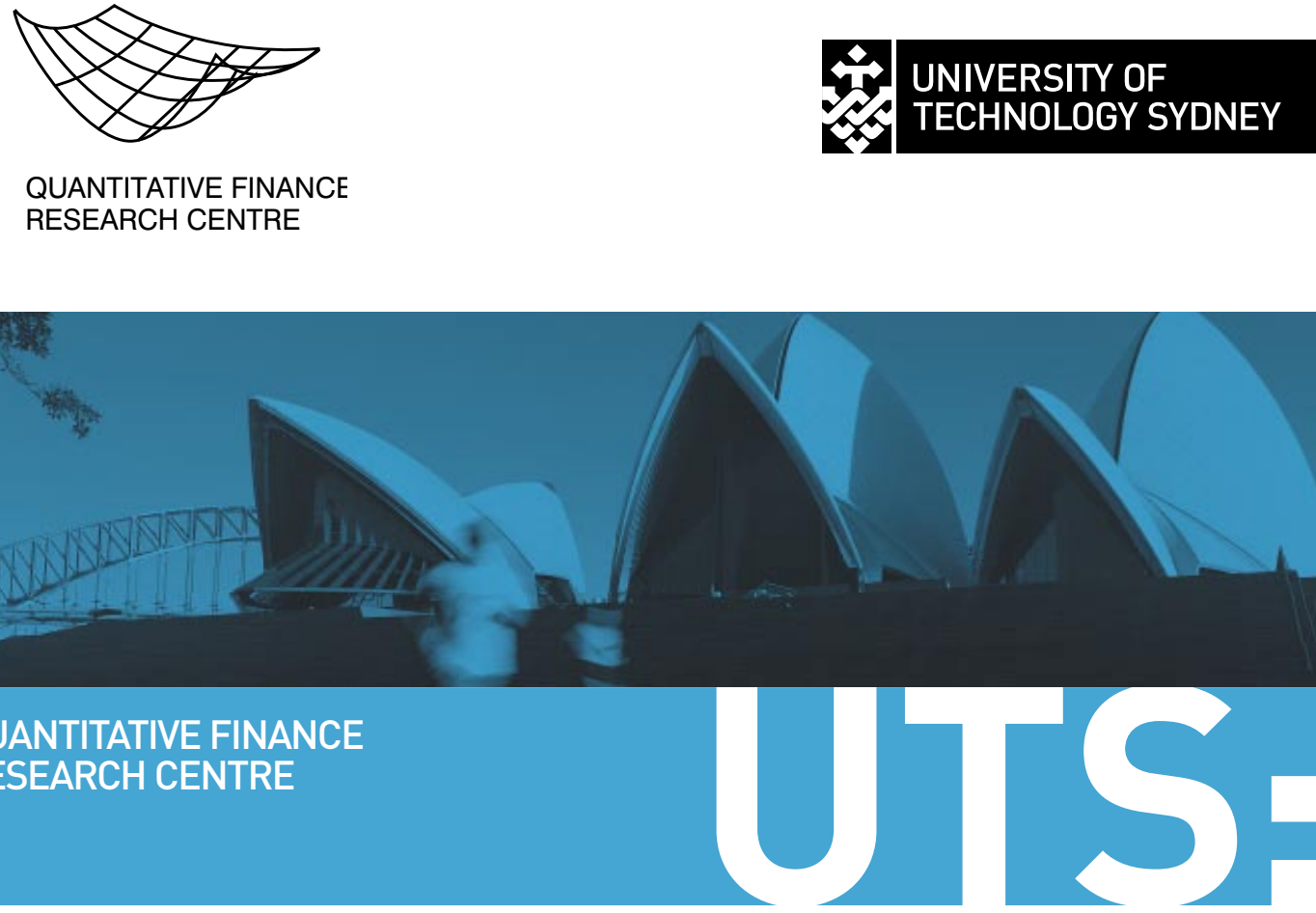

THINK.CHANGE.DO

Quantitative Finance Research Centre

Research Paper 169

October 2005

Parameterizing Unconditional Skewness in Models for Financial Time Series

Changli He, Annastiina Silvennoinen and Timo Teräsvirta 


\title{
Parameterizing unconditional skewness in models for financial time series
}

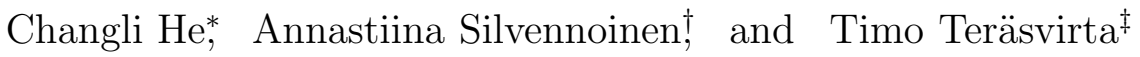 \\ Department of Economic Statistics, Stockholm School of Economics, \\ P. O. Box 6501, SE-113 83 Stockholm, Sweden
}

December 2005

\begin{abstract}
In this paper we consider the third-moment structure of a class of nonlinear time series models. It is often argued that the marginal distribution of financial time series such as returns is skewed. Therefore it is of importance to know what properties a model should possess if it is to accommodate unconditional skewness. We consider modelling the unconditional mean and variance using models that respond nonlinearly or asymmetrically to shocks. We investigate the implications of these models on the third-moment structure of the marginal distribution as well as conditions under which the unconditional distribution exhibits skewness and nonzero third-order autocovariance structure. In this respect, an asymmetric or nonlinear specification of the conditional mean is found to be of greater importance than the properties of the conditional variance. Several examples are discussed and, whenever possible, explicit analytical expressions provided for all third-order moments and cross-moments. Finally, we introduce a new tool, the shock impact curve, for investigating the impact of shocks on the conditional mean squared error of return series.
\end{abstract}

JEL classification: C22

Key words: Asymmetry; GARCH; Nonlinearity; Shock Impact Curve; Time series; Unconditional skewness;

\footnotetext{
*e-mail: changli.he@hhs.se

$\dagger$ e-mail: annastiina.silvennoinen@hhs.se

${ }^{\ddagger}$ e-mail: timo.terasvirta@hhs.se

Acknowledgements. This research has been supported by the Jan Wallander's and Tom Hedelius's Foundation, Grant No. J03-41. We would like to thank Pentti Saikkonen, Markku Lanne, Tony Hall, Peter Phillips, and Mika Meitz for useful discussions. Part of the research was done while the second and the third author were visiting the School of Finance and Economics, University of Technology, Sydney, whose kind hospitality is gratefully acknowledged. Our very special thanks go to Tony Hall for making the visit possible. Material from this paper has been presented at the workshop 'Econometrics and Computational Economics', Helsinki, November 2004, 14th meeting of the New Zealand Econometric Study Group, Christchurch, March 2005, RUESG Workshop on Financial Econometrics, Helsinki, August 2005, International Conference on Finance, Copenhagen, September 2005, and in seminars at Stockholm School of Economics, and the University of Technology, Sydney. We would like to thank the participants in these occasions for their comments. The responsibility for any errors and shortcomings in this paper remains ours.
} 


\section{Introduction}

Financial series such as high-frequency asset returns have little forecastable structure in the mean. For this reason, and because volatility is used as a measure of risk, forecasting volatility and thus modelling the conditional variance has been the main concern of practitioners. The most popular family of volatility models, the GARCH family, see Bollerslev (1986) for the standard GARCH model, is used to characterize two important stylized facts of return series: fat tails of the marginal distribution of returns and volatility clustering, that is, higher-order dependence observed in the series.

Another feature of these series that has attracted attention is an asymmetric response of volatility to shocks. GARCH models that can take this into account include the Threshold GARCH (Zakoïan, 1994), the GJR-GARCH (Glosten, Jagannathan, and Runkle, 1993), and the Smooth Transition GARCH (Hagerud, 1997; González-Rivera, 1998) model. Pagan and Schwert (1990) and Engle and $\mathrm{Ng}$ (1993) have suggested a practical way of describing this response by the so-called News Impact Curve (NIC).

In addition, it has been observed that the marginal distribution of returns is sometimes skewed. Harvey and Siddique (1999), Chen, Hong, and Stein (2001), and Engle and Patton (2001), to mention a few contributions, report evidence for financial time series with asymmetric distributions. However, as pointed out by Peiro (2002, 2004), one should not go as far as stating the skewness of marginal distributions of returns as a stylized fact, nor rely solely on its traditional measurement under normality. One should investigate possible asymmetry of the distribution using not only traditional tests but distribution-free measurements as well, see also Kim and White (2004). Attempts to model this skewness through defining the concept of conditional skewness have been made, see for instance Harvey and Siddique (1999), Lambert and Laurent (2002) and references therein, Brännäs and Nordman (2003a), Brännäs and Nordman (2003b), and Harris, Küçüközmen, and Yilmaz (2004). This requires giving up a standard assumption in econometric work, namely, that noise sent through a parametric filter to generate the output has a symmetric distribution around zero. (Of course, modelling positive-valued series constitutes an exception.) Furthermore, in some cases, see Hansen (1994), one even gives up the assumption, otherwise invariably made in the context of GARCH processes, that the errors of the process are independent.

It may be conceptually difficult to understand why the noise that in principle should contain no information about the properties of the process should have a non-symmetric distribution and thus be informative about the output. For this reason, in this paper we make an effort to find out under which conditions the marginal distribution of returns can be skewed while the noise has a symmetric distribution. It turns out that for this purpose we have to study processes with a nonconstant conditional mean. In so doing, we shall be interested in the case where a shock can have a nonzero effect on both the conditional mean and conditional variance. This leads us to consider processes with a symmetric or asymmetric, and linear or nonlinear conditional mean. There is empirical evidence of some return series having an asymmetric conditional mean; see Brännäs and de Gooijer (2004). An example of a nonlinear conditional mean is the wellknown GARCH-in-mean (GARCH-M) process introduced by Engle, Lilien, and Robins (1987). Lanne and Saikkonen (2005) recently considered a GARCH-M model with an asymmetric error distribution. These asymmetries and nonlinearities are likely to affect the marginal distribution of the series to be modelled but, to the best of our knowledge, their effect on the third-moment structure of the process has not been investigated.

The starting-point of the present paper is a model whose first two conditional moments are parametric and the error distribution is symmetric around zero. The asymmetric moving 
average (asMA) model by Wecker (1981) serves as an example of such a model. It nests a linear moving average model, and for this reason the effect of the asymmetry in the conditional mean on the third-moment structure of the process can be easily investigated by imposing appropriate parameter restrictions on the model. Examining the role of the conditional mean as a whole in this framework is quite straightforward. Our other example will be the GARCH-M model. It is well known (Hong, 1991) that the GARCH-M model implies autocorrelated returns, but it is probably less well known that introducing a function of the conditional variance in the conditional mean makes the marginal distribution of the observations skewed.

For the purpose of deriving analytical expressions for unconditional third-order moments, parameterizing the conditional standard deviation is preferable to parameterizing the conditional variance. In the latter case, the definitions of moments would involve expectations that do not have analytic expressions. For this reason, we focus on the threshold GARCH (TGARCH) model that in turn nests the absolute-value GARCH (AVGARCH) model of Taylor (1986) and Schwert (1989). The TGARCH model has an asymmetric response to shocks, whereas the same response in the AVGARCH model is symmetric as it is in the standard GARCH model of Bollerslev (1986). General conclusions drawn from these two models of the conditional standard deviation are applicable to other GARCH models as well.

Recently, Brännäs and de Gooijer (2004) proposed a model that introduces asymmetry both in the conditional mean and the conditional variance. The variance is an extension of the QGARCH model of Sentana (1995). The authors considered the first and second moments of their asMA-asGARCH model but did not investigate the third-moment structure of their model. Because they parameterize the conditional variance, not the conditional standard deviation, finding analytical expressions for the third-order moments appears difficult. In fact, it seems that even lower-order moments may not have analytical expressions readily available. As already suggested, general conclusions from our models will be applicable to the asMA-asGARCH model.

It turns out that there is a rather large set of asMA-TGARCH parameter values such that the marginal distribution of the observations will be skewed. Not all of them are relevant in the sense that they would correspond to situations experienced in practice. For example, we may not expect the volatility to respond more strongly to positive than it does to negative shocks of the same size. In order to study the relevance of the parameter combinations in question we generalize the News Impact Curve (NIC) of Engle and Ng (1993) in order to account for the structure in the conditional mean. For this purpose we define a new concept, the Shock Impact Curve (SIC), that describes the impact of a shock on the conditional mean squared error of the series, and apply it for our purposes.

The paper is organized as follows. The general model is introduced in Section 2 and its moment structure up to the third moments derived in Section 3. Special cases are presented in Section 4. The shock impact curve is defined and applied in Section 5. Conclusions from this study can be found in Section 6. Technical derivations and expressions of the moments are contained in the Appendix.

\section{The model family}

Let $y_{t}$ be generated by

$$
\begin{aligned}
y_{t} & =\mu_{t}+\varepsilon_{t}, \\
\varepsilon_{t} & =z_{t} h_{t}
\end{aligned}
$$


where $\mu_{t}$ is the conditional mean of $y_{t}$ given $\mathcal{F}_{t-1}$ (the sigma-field generated by the available information until time $t-1), h_{t}^{2}$ is the conditional variance of $y_{t}$ given $\mathcal{F}_{t-1}$, and $\left\{z_{t}\right\} \sim i i d(0,1)$ with a distribution function that is symmetric around zero. The processes $\mu_{t}$ and $h_{t}$ are measurable with respect to $\mathcal{F}_{t-1}$.

We consider a variety of examples from two classes of models for the conditional mean and especially focus on the ability of these models to exhibit asymmetric or nonlinear behaviour. For simplicity, we focus on first-order models which are empirically often found to be adequate. The equation

$$
\mu_{t}=\phi \varepsilon_{t-1}+\phi^{+}\left(\varepsilon_{t-1}^{+}-E \varepsilon_{t}^{+}\right)
$$

where $\varepsilon_{t}^{+}=\max \left(0, \varepsilon_{t}\right)$, defines the first-order asymmetric moving average (asMA) process of Wecker (1981). For $\phi^{+} \neq 0$ the model is asymmetric and linear in its response to shocks. Note that model (3) nests an MA process which is symmetric and linear. If $\mu_{t}$ is a function of $h_{t}$ such that

$$
\mu_{t}=\phi\left(h_{t}^{\delta}-E h_{t}^{\delta}\right), \quad \delta=1 \text { or } 2
$$

we have the GARCH-in-mean (GARCH-M) model of Engle, Lilien, and Robins (1987). In this case the model for the conditional mean is nonlinear and the degree of asymmetry is controlled by the asymmetry of the GARCH process.

The error process $\left\{\varepsilon_{t}\right\}$ of (1) is assumed to be a conditionally heteroskedastic white noise sequence with

$$
h_{t}^{d}=\omega+c_{t-1} h_{t-1}^{d}, \quad d=1 \text { or } 2
$$

where $c_{t}=c_{t}\left(z_{t}\right)$ is a well-defined function and $h_{t}^{d}>0$ for all $t$. To ensure this, suitable parameter restrictions must be imposed. The moment properties of the family of GARCH models defined by (5) are investigated in He and Teräsvirta (1999). It nests many of the models in the family of GARCH models of Hentschel (1995). For instance, setting $d=2$ in (5) and

$$
c_{t}=\alpha z_{t}^{2}+\beta
$$

yields the standard GARCH model of Bollerslev (1986). Setting $d=1$ and

$$
c_{t}=\alpha\left|z_{t}\right|+\beta+\alpha^{*} z_{t}
$$

equation (5) defines the threshold GARCH (TGARCH) model of Zakoïan (1994). By setting $\alpha^{*}=0$, the model collapses into the AVGARCH model of Taylor (1986) and Schwert (1989). Furthermore, the GJR-GARCH model of Glosten, Jagannathan, and Runkle (1993) and the nonlinear GARCH (NLGARCH) model of Engle (1990) are nested in (5). Note that any GARCH model defined by equation (5) is symmetric in its response to shocks if and only if $c_{t}\left(z_{t}\right)$ in (5) is an even function of $z_{t}$. The following theorem states conditions under which the process $\left\{\varepsilon_{t}\right\}$ in (2) and (5) is strictly and $m d$-order stationary.

Theorem 1 If $E\left|z_{t}^{\lambda d}\right|<\infty$ and $E c_{t}^{\lambda}<1$ for some $\lambda \in(0,1]$, then there exists a unique $\lambda d$ order stationary solution to (2) and (5). The solution is strictly stationary and ergodic. If $E\left|z_{t}^{d m}\right|<\infty$, then the necessary and sufficient condition for the existence of the mdth moment of the solution $\left\{\varepsilon_{t}\right\}$ in (2) is $E c_{t}^{m}<1$ where $m$ is a positive integer.

For a proof, see Theorems 2.1 and 2.2 in Ling and McAleer (2002). 


\section{Moments}

We begin by considering the moment structure of the general model (1) and (2). Assume that $\left\{y_{t}\right\}$ is strictly stationary with finite third-order moments and set $\gamma_{i}=E\left(y_{t}-E y_{t}\right)^{i}$ and $\gamma_{i j}(k)=\operatorname{Cov}\left(y_{t}^{i}, y_{t-k}^{j}\right), i, j \geq 1$. The unconditional mean and variance are $E y_{t}=E \mu_{t}$ and $\gamma_{2}=\operatorname{Var} \mu_{t}+E \varepsilon_{t}^{2}$, respectively. The autocovariances are $\gamma_{11}(k)=\operatorname{Cov}\left(\mu_{t}, \mu_{t-k}\right)+E \mu_{t} \varepsilon_{t-k}$ where $k \geq 1$. Assuming $\mu_{t}$ is an asMA process in (3) and $\phi^{+} \neq 0$ renders the autocovariances nonzero for $k \geq 1$, see Lemma 1 in the Appendix. The same holds if $\mu_{t}$ is a function of $h_{t}^{\delta}, \delta=1$ or 2 , see Lemma 3.

The third moment and third-order cross-moments of $y_{t}$ are given by

$$
\begin{aligned}
\gamma_{3} & =E\left(\mu_{t}-E \mu_{t}\right)^{3}+3 \operatorname{Cov}\left(\mu_{t}, \varepsilon_{t}^{2}\right) \\
\gamma_{21}(k) & =\operatorname{Cov}\left(\mu_{t}^{2}, \mu_{t-k}\right)+\operatorname{Cov}\left(\mu_{t-k}, \varepsilon_{t}^{2}\right)+E \mu_{t}^{2} \varepsilon_{t-k}+E \varepsilon_{t}^{2} \varepsilon_{t-k}, \quad k \geq 1 \\
\gamma_{12}(k) & =\operatorname{Cov}\left(\mu_{t}, \mu_{t-k}^{2}\right)+\operatorname{Cov}\left(\mu_{t}, \varepsilon_{t-k}^{2}\right)+2 E \mu_{t} \mu_{t-k} \varepsilon_{t-k}, \quad k \geq 1
\end{aligned}
$$

Define the unconditional skewness of $y_{t}$ as $\kappa_{3}=\gamma_{3} /\left(\gamma_{2}\right)^{3 / 2}$. The following proposition gives general conditions that yield zero skewness.

Proposition 2 Consider the model in (1) and (2) that is third-order stationary. The conditions $E\left(\mu_{t}-E \mu_{t}\right)^{3}=0$ and $\operatorname{Cov}\left(\mu_{t}, \varepsilon_{t}^{2}\right)=0$ are sufficient for $\kappa_{3}=0$.

When $\mu_{t} \equiv \mu$ (constant) in (1), the conditions in Proposition 2 are satisfied and thus $\gamma_{3}=$ 0 . In this case, the only nonzero cross-moments are $\gamma_{21}(k)=E \varepsilon_{t}^{2} \varepsilon_{t-k}, k \geq 1$. Therefore, only assuming that the conditional second moment is time-varying does not imply nonzero unconditional skewness. A time-varying conditional mean is required for that. ${ }^{1}$

\section{Examples}

In this section we consider two specifications of the conditional mean in detail. The first one is the first-order asymmetric moving average model of Wecker (1981). The second one (GARCH-M) introduces the conditional standard deviation or variance into the conditional mean. We choose the TGARCH model for the error process $\left\{\varepsilon_{t}\right\}$. This choice is dictated by our goal which is to obtain analytical expressions for all unconditional third-order moments and cross-moments. Such expressions give an idea of how asymmetries and nonlinearities in conditional first and second moments contribute to the unconditional third moments. Since the TGARCH model is asymmetric in its response to shocks and nests the symmetric AVGARCH model, it is possible to isolate the effect of this asymmetry on the unconditional skewness. Analytical expressions for the moments can be found in Lemma 2 and the subsequent Corollaries and in Lemma 4 of the Appendix. These expressions are rather involved but yield quite straightforward conclusions.

Other GARCH models are likely to be similar to the TGARCH model in this respect but because most of them lack analytical expressions for third-order moments, one has to rely on simulations to obtain numerical values for them. ${ }^{2}$ Whenever possible, we try to take examples of models such as the symmetric GARCH model of Bollerslev (1986), the asymmetric QGARCH

\footnotetext{
${ }^{1}$ One motivation for extending standard symmetric GARCH models to include the leverage effect has been to create asymmetric unconditional densities, see e.g. Lambert and Laurent (2002). Engle and Patton (2001) also write that 'the asymmetric structure of volatility generates skewed distributions of forecast prices'.

${ }^{2}$ The possibility of using quantile measures as in Kim and White (2004) for unconditional skewness is yet to be explored.
} 
one of Sentana (1995), or the GJR-GARCH model of Glosten, Jagannathan, and Runkle (1993). In fact, the results in the Appendix apply to the general family of GARCH models (5). However, fully explicit expressions for third moments are provided only for the TGARCH or the AVGARCH model and not for models for which $d=2$ in (5). Note that the QGARCH model is not a member of the family defined by equation (5).

We begin by considering the complete asMA-TGARCH model and the effect of restricting the conditional standard deviation to be a symmetric AVGARCH model. Subsequently, we consider the third-moment structure of the model when the conditional mean is simplified to only contain either the asymmetric component $(\phi=0)$, the symmetric MA component $\left(\phi^{+}=0\right)$, or neither $\left(\phi=\phi^{+}=0\right)$. In all these cases we consider both the TGARCH and the AVGARCH specifications for the conditional second moment. As a final example, the conditional mean is defined to be a function of the conditional second moment, which leads us to GARCH-M models.

\subsection{First-order asMA model with TGARCH or AVGARCH conditional stan- dard deviation}

The third-moment structure of the first-order asMA-TGARCH model is characterized by Lemmas 2 and 5. It is apparent from the rather involved expressions that this model accommodates a rich variety of third-order moment structures.

For an asMA process combined with a model for conditional heteroskedasticity the autocovariances $\gamma_{11}(k)$ are nonzero for all lags $k \geq 0$, as also pointed out in Brännäs and de Gooijer (2004). This is the case for both symmetric and asymmetric GARCH processes. After a peak at the first lag, the values of autocorrelation are very low and the decay rate is slow. Brännäs and de Gooijer (2004) claim that it may be empirically possible to discriminate between an $\operatorname{asMA}(q)$ model with a constant conditional variance and one with a GARCH process for the conditional variance because of the difference in autocovariances. The former process has nonzero autocovariances up until lag $q$, and zero thereafter, whereas the latter has nonzero autocovariances for all lags. However, at least for the first-order asMA-GARCH model the autocovariances are very close to zero after first lag, which complicates distinguishing between them this way.

Since the expressions for the third-order moments are quite involved we illustrate the situation numerically. The following figures are produced using the standardized Gaussian error distribution. Figure 1 shows the amount of unconditional skewness that can be obtained from an invertible asMA-TGARCH process for certain parameter values of the conditional mean. Invertibility of the asMA process has to be checked using simulations, see e.g. Brännäs and de Gooijer (1995). Each curve represents the level of unconditional skewness for a fixed value of $\phi^{+}$and a suitable range of values for $\phi$. When $|\phi|$ is large, the invertibility condition restricts the asymmetry parameter $\phi^{+}$and thus limits the achievable amount of skewness.

When the conditional standard deviation is restricted to follow the AVGARCH model, some of the expressions in Lemma 2 simplify, but not substantially. The resulting moment structure is given in Corollary 1. In this case the cross-moments $E \varepsilon_{t}^{2} \varepsilon_{t-k}, E \varepsilon_{t-k} \varepsilon_{t}^{+}, E \varepsilon_{t-k} \varepsilon_{t}^{+2}$, $E \varepsilon_{t-k} \varepsilon_{t-k-1} \varepsilon_{t}^{+}$, and $E \varepsilon_{t-k} \varepsilon_{t}^{+} \varepsilon_{t-k-1}^{+}, k \geq 1$, equal zero, which can be seen from the expressions in Lemma 5 and Corollary 5. Thus the third-moment structure is still rich as long as the conditional mean is defined by an asMA model. In particular, all third-order moments are nonzero, whether or not the conditional standard deviation exhibits asymmetry. However, there is a reduction on the amount of skewness when the conditional standard deviation is no longer asymmetric, which is seen by comparing the top and bottom panels in Figure 1. What seems 
to have an even larger effect is the increase in the persistence of the GARCH process, which increases the skewness of the marginal distribution whenever $\phi^{+} \neq 0$.

Next consider the case where $\phi=0$. It can be seen from the expressions in Corollary 2 that the third-moment structure is still rather rich and complex. It is, however, considerably simpler than in the case of $\phi \neq 0$. When the errors are restricted to follow a symmetric AVGARCH model, the expressions simplify somewhat, see Corollary 3, but again not very much. Expressions in Lemma 5 and Corollary 5 show that $E \varepsilon_{t}^{2} \varepsilon_{t-k}=E \varepsilon_{t-k} \varepsilon_{t}^{+}=E \varepsilon_{t-k} \varepsilon_{t}^{+2}=E \varepsilon_{t-k} \varepsilon_{t}^{+} \varepsilon_{t-k-1}^{+}=0$, $k \geq 1$. Thus, regardless of the conditional standard deviation, an asymmetric conditional mean leads to a skewed marginal distribution for $y_{t}$ and nonzero third-order cross-moments. An obvious conclusion is that asymmetry of the conditional mean plays a very influential role in determining both the sign and the amount of skewness in this distribution.

\subsection{First-order MA model with TGARCH or AVGARCH conditional stan- dard deviation}

A rather simple third-moment structure follows when $\phi^{+}=0$ in (3) while the conditional standard deviation follows the TGARCH model. This is evident from the results in Corollary 4. In this case we still have $E y_{t}^{3} \neq 0$. An interesting feature is that $E y_{t}^{2} y_{t-k} \neq 0, k \geq 1$, whereas $E y_{t} y_{t-k}^{2}=0$ for $k>1$. It should also be noted that the only nonzero cross-moment of $\varepsilon_{t}$ is now $E \varepsilon_{t}^{2} \varepsilon_{t-k}$. In fact, $\kappa_{3}=0$ if and only if $E \varepsilon_{t}^{2} \varepsilon_{t-1}=0$, Furthermore, also assuming $\phi=0$ in (3), i.e. having $\mu_{t}=0$, forces the unconditional skewness to zero regardless of the asymmetry in the conditional second moment. In this case the only nonzero cross-moments are $E y_{t}^{2} y_{t-k}=E \varepsilon_{t}^{2} \varepsilon_{t-k}$, $k \geq 1$. These results may be useful in specifying asMA-TGARCH models.

The thick curve in Figure 1 represents the skewness as a function of $\phi$ for $\phi^{+}=0$. In the top panels it intersects the $x$-axis at $\phi=0$ in accordance with the results mentioned after Proposition 2.

As an aside consider a model whose conditional mean specification is a first-order AR process: $\mu_{t}=\phi y_{t-1}$ in (1). In this case

$$
\kappa_{3}=\frac{3 \sum_{k=1}^{\infty} \phi^{i} E \varepsilon_{t}^{2} \varepsilon_{t-k} /\left(1-\phi^{3}\right)}{\left(E h_{t}^{2} /\left(1-\phi^{2}\right)\right)^{3 / 2}} .
$$

Clearly $\kappa_{3}=0$ if and only if $E \varepsilon_{t}^{2} \varepsilon_{t-k}=0$ for all $k \geq 1$. The unconditional skewness emerging from this model is very similar to that of the MA-TGARCH model already discussed. In Figure 2 the unconditional skewness is plotted as a function of the mean parameter $\phi$ for a range of values for $\beta$ and keeping the other TGARCH parameter values fixed. It can be concluded that the amount of skewness obtained from a model with a linear and symmetric conditional mean is not large and the effect of increasing persistence in the GARCH process on skewness is negligible.

We now turn to the analytic form of the cross-moment $E \varepsilon_{t}^{2} \varepsilon_{t-k}$ in Corollary 4 . When the conditional standard deviation is defined as a TGARCH process, it follows that

$$
E \varepsilon_{t}^{2} \varepsilon_{t-k}=2 \omega \alpha^{*} E h_{t}^{2} \sum_{j=0}^{k-1}\left(E c_{t}\right)^{k-1-j}\left(E c_{t}^{2}\right)^{j}+2 \alpha^{*}\left(E c_{t}^{2}\right)^{k-1} E c_{t}\left|z_{t}\right|^{2} E h_{t}^{3}, \quad k \geq 1
$$

where the expressions for the moments of $c_{t}$ and $h_{t}$ are given in Lemma 5 . Hence $E \varepsilon_{t}^{2} \varepsilon_{t-k} \neq 0$ for $\alpha^{*} \neq 0$. Assuming $\alpha^{*}=0$ yields $E \varepsilon_{t}^{2} \varepsilon_{t-k}=0, k \geq 1$, which implies that the third moment and all the third-order cross-moments in Corollary 4 are zero. In fact, any parameterization of $h_{t}$ or $h_{t}^{2}$ that has the property $E \varepsilon_{t}^{2} \varepsilon_{t-k}=0$ for $k \geq 1$ gives the same result. As stated 
Figure 1: asMA-TGARCH: Unconditional skewness of $y_{t}$ as a function of $\phi$ for the following values of $\phi^{+}$ and the TGARCH parameters: lines: $\phi^{+}=1.0,0.75, \ldots,-1.0$ (top to bottom), thick line corresponds to $\phi^{+}=0$; TGARCH parameters: $\omega=0.005, \alpha=0.05, \beta=0.90$ (left-hand panels), $\beta=0.94$ (right-hand panels), $\alpha^{*}=-0.04$ (top panels), and $\alpha^{*}=0$ (bottom panels).
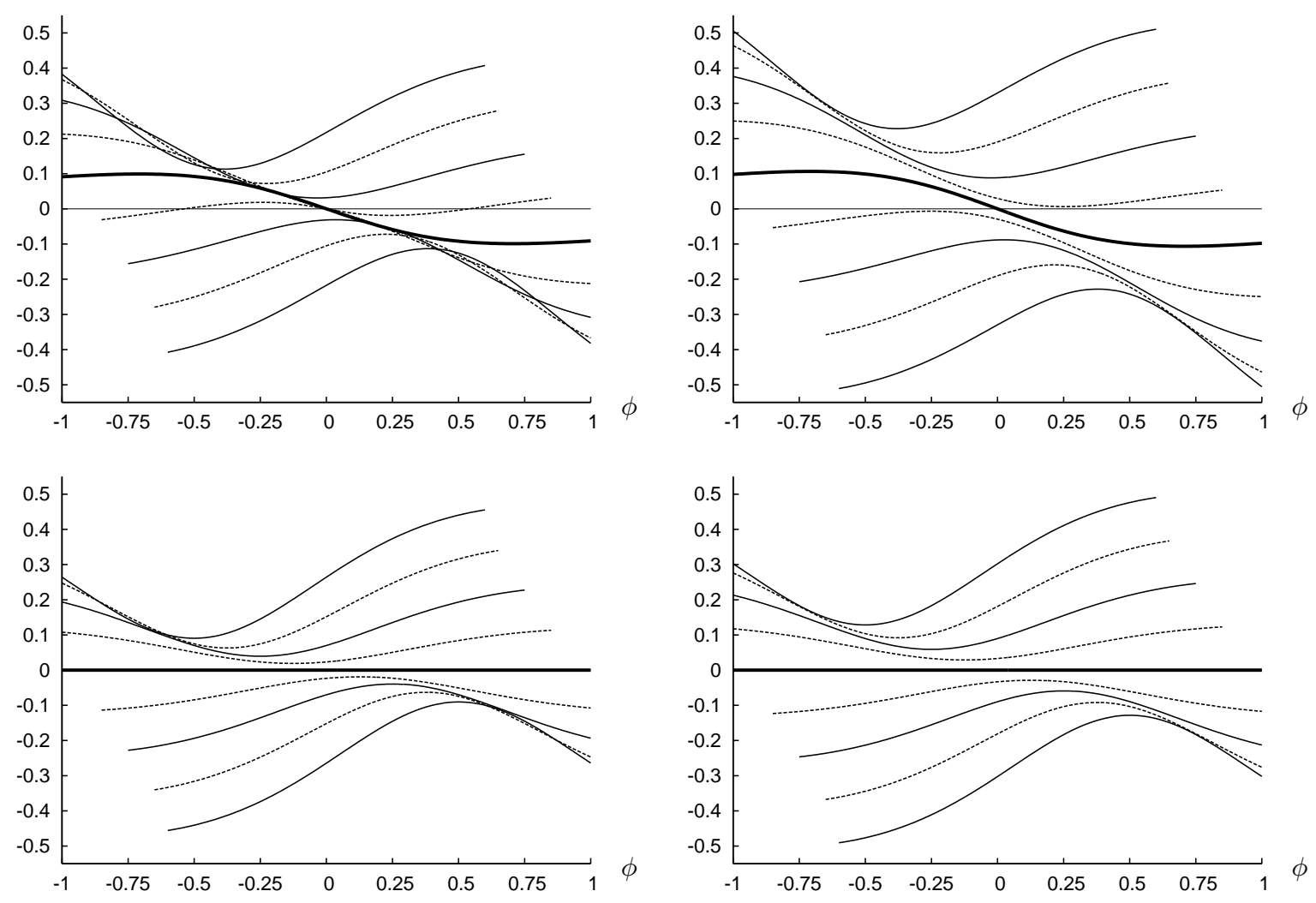

in Corollary 5, all symmetric GARCH models belonging to the family (5) have that property. The standard GARCH model of Bollerslev (1986) is an example of such a case. As further examples, consider the nonlinear models where the errors are governed by a first-order QGARCH process $h_{t}^{2}=\omega+\alpha \varepsilon_{t-1}^{2}+\beta h_{t-1}^{2}+\alpha^{*} \varepsilon_{t-1}$ (Sentana, 1995) or by a GJR-GARCH process $h_{t}^{2}=$ $\omega+\alpha \varepsilon_{t-1}^{2}+\beta h_{t-1}^{2}+\alpha^{*} \varepsilon_{t-1}^{+2}$ (Glosten, Jagannathan, and Runkle, 1993). If the errors follow a QGARCH process then the expression for $E \varepsilon_{t}^{2} \varepsilon_{t-k}$ is given by

$$
E \varepsilon_{t}^{2} \varepsilon_{t-k}=\alpha^{*}(\alpha+\beta)^{k-1} E h_{t}^{2}, \quad k \geq 1,
$$

where

$$
E h_{t}^{2}=\frac{\omega}{1-(\alpha+\beta)} .
$$

If the errors follow a GJR-GARCH process, $d=2$ and $c_{t}=\alpha z_{t}^{2}+\beta+\alpha^{*} z_{t}^{+2}$ in (5),

$$
E \varepsilon_{t}^{2} \varepsilon_{t-k}=\alpha^{*}\left(\alpha+\beta+\alpha^{*} E z_{t}^{+2}\right)^{k-1} E z_{t}^{+3} E h_{t}^{3}, \quad k \geq 1 .
$$

An explicit expression for $E h_{t}^{3}$ is not available but is known not to be trivially zero. Also in these cases, $E \varepsilon_{t}^{2} \varepsilon_{t-k} \neq 0$ if and only if $\alpha^{*} \neq 0$. Thus, if the conditional mean is symmetric and linear, and the conditional second moment is symmetric, the unconditional marginal distribution for $y_{t}$ is symmetric around zero. In the bottom panels of Figure 1, the thick line corresponding 
Figure 2: AR-TGARCH: Unconditional skewness of $y_{t}$ as a function of $\phi$ for the following values of the TGARCH parameters: $x$-axis: $\phi, y$-axis: unconditional skewness, $\omega=0.005, \alpha=0.05, \beta=0.94$ (solid line), $\beta=0.90$ (dashed line), $\alpha^{*}=0.04$ (left), $\alpha^{*}=0$ (middle), and $\alpha^{*}=-0.04$ (right).
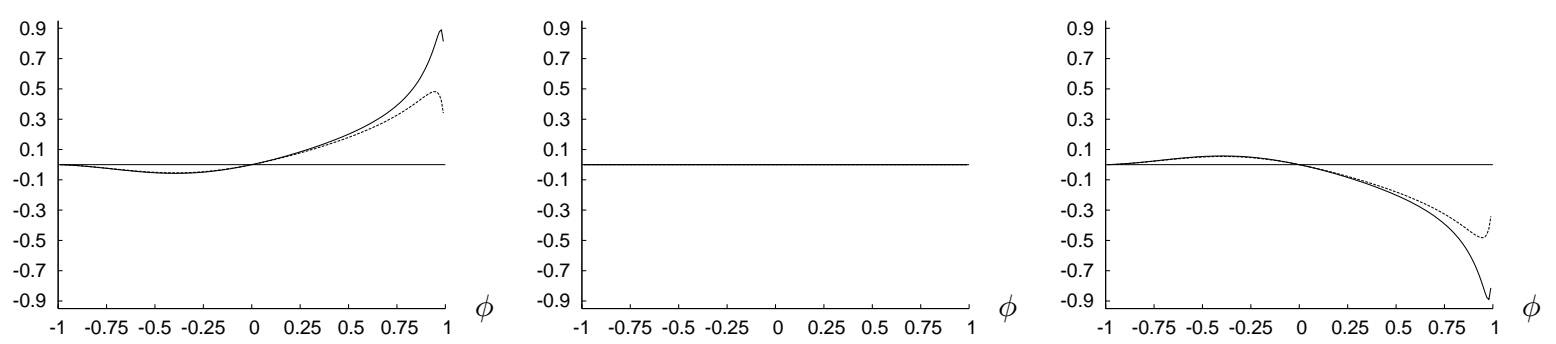

to $\phi^{+}=0$ illustrates this finding. It is emphasized that the results just discussed are only obtained if $\phi \neq 0$. Thus, at least some linear dependence in $\left\{y_{t}\right\}$ is necessary for skewness in the unconditional distribution of $y_{t}$.

We may also note that in the case of the first-order asQGARCH model $h_{t}^{2}=\omega+\alpha \varepsilon_{t-1}^{2}+$ $\beta h_{t-1}^{2}+\alpha^{*} \varepsilon_{t-1}+\alpha^{* *} \varepsilon_{t-1}^{+}$(Brännäs and de Gooijer, 2004), the expression for $E \varepsilon_{t}^{2} \varepsilon_{t-k}$ becomes very complicated, and it does not seem possible to derive an analytical form for it. However, this moment seems to be nonzero for $k \geq 1$ because its components are not trivially zero. ${ }^{3}$

\subsection{GARCH-in-mean model}

It is well known that when the conditional standard deviation, conditional variance or any other nontrivial function of these, enters the conditional mean, $\gamma_{11}(k) \neq 0$ for $k \geq 1$ if $\gamma_{2}<\infty$; see Hong (1991). It may be less well known that in this case $\gamma_{3} \neq 0$. As an example, consider the TGARCH-M model (1),(2), and (4)-(6) so that $E y_{t}=0$. Since $h_{t}^{\delta}$ is a positive-valued variable and its distribution is asymmetric, it follows that $E y_{t}^{3} \neq 0$. The third moment and third-order cross-moments of the third-order stationary TGARCH-M process are given by Lemma 4 . The unconditional skewness from a TGARCH-M model with $\delta=1$ and $\delta=2$ are plotted in Figure 3 as a function of $\phi$. The figure shows that the range of possible skewness increases with the persistence of the GARCH process. It is also seen that when the conditional standard deviation enters the conditional mean, the distribution becomes more skewed than it would be if the conditional mean were a function of the conditional variance. Assuming $\phi=0$ implies $\gamma_{3}=0$ regardless of any asymmetry in the conditional standard deviation or conditional variance. This is also seen from Figure 3 where all the lines intersect the $x$-axis at $\phi=0$. In this case the only nonzero cross-moments are $E y_{t}^{2} y_{t-k}=E h_{t}^{2} \varepsilon_{t-k}$; see the discussion in the previous subsection.

Assuming that $h_{t}$ is defined by the standard AVGARCH model, the expressions for the moments simplify somewhat. Then $E h_{t}^{2} \varepsilon_{t-k}=E h_{t} \varepsilon_{t-k}=E h_{t} h_{t-k} \varepsilon_{t-k}=0, k \geq 1$, as can be seen from the expressions in Lemma 5 and Corollary 5 . However, provided that $\phi \neq 0$, all third-order moments are nonzero regardless of whether the conditional standard deviation is symmetric or asymmetric. The amount of skewness in this case is considerably less than in the case of the TGARCH-M model, which can be seen by comparing the top and bottom panels of Figure 3.

This example demonstrates that the third-moment structure in the case of the TGARCH-M or AVGARCH-M model is richer than it is in MA-TGARCH and MA-AVGARCH models, respectively. It can be concluded that both asymmetric and nonlinear responses to shocks in

\footnotetext{
${ }^{3}$ In fact, it seems that there is no explicit expression for any moment $E \varepsilon_{t}^{m}, m>1$, for this model - at least it seems that there does not exist an analytic form for $E h_{t}^{2}$.
} 
Figure 3: TGARCH-M: Unconditional skewness of $y_{t}$ as a function of $\phi$ for the following values of $\delta$ and the TGARCH parameters: $\delta=1$ (left-hand panels) and $\delta=2$ (right-hand panels), TGARCH parameters: $\omega=0.005, \alpha=0.05, \beta=0.94$ (solid line), $\beta=0.90$ (dashed line), $\alpha^{*}= \pm 0.04$ (top panels), and $\alpha^{*}=0$ (bottom panels).
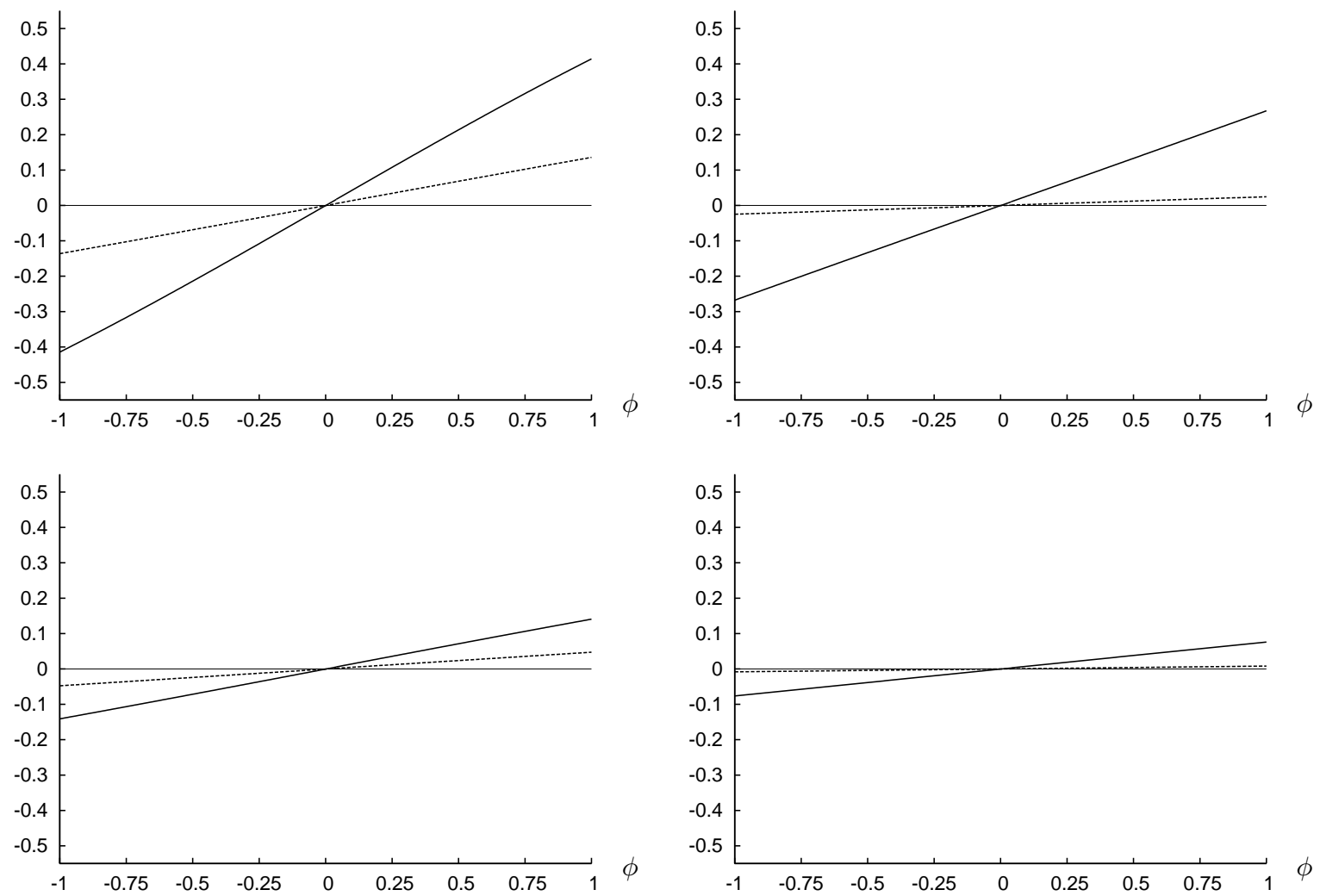

the conditional mean play an important role in producing skewness in the marginal density of $y_{t}$.

\section{$5 \quad$ Shock impact curves}

Engle and $\mathrm{Ng}$ (1993) defined the news impact curve as a function that describes the impact of a shock $\varepsilon_{t-1}$ on current volatility expressed as conditional variance $h_{t}^{2}$. The shock is the component of the return $y_{t}$ that can be characterized as 'news' to the agents in the following model:

$$
y_{t}=f\left(y_{t-j}, \varepsilon_{t-j} ; j \geq 1\right)+\varepsilon_{t} .
$$

In this model, the conditional mean $E_{t-1} y_{t}$ is not constant over time but is a function of past shocks. It is assumed that the conditional mean component is not news but rather structure known to the agents. For this reason, the NIC is measuring the impact of a shock on the conditional variance of the return. Nevertheless, for the purposes of this paper it will be useful to introduce a slight extension that also involves the shock coming through the conditional mean. It is called the Shock Impact Curve (SIC) and describes the impact of the shock on the conditional mean squared error of the return. The SIC is defined as follows:

$$
E_{t-1}^{\mathrm{SIC}} y_{t}^{2}=\mu_{t}^{2}(\sigma)+h_{t}^{2}(\sigma)
$$


where $\mu(\sigma)$ and $h^{2}(\sigma)$ are the conditional mean and variance with elements in $\mathcal{F}_{t-2}$ replaced with their unconditional counterparts, for instance $\operatorname{Var}_{t-k-1} y_{t-k}=h_{t-k}^{2}, k \geq 1$, is replaced with $\sigma^{2} \stackrel{\text { def }}{=} \operatorname{Var} y_{t}=E \mu_{t}^{2}+E h_{t}^{2}$. It may be argued that the correlation structure of $\left\{y_{t}\right\}$ is known to the agents, whereby that part of the response does not qualify as news. If this structure is weak, however, it may be difficult in practice to separate this effect from the actual 'news'. If $\mu_{t}=0$, SIC coincides with NIC. Conversely, the impact of 'news' and that of a 'shock' on the next return can have rather different shapes.

The SIC can be used to study the effect of a shock on both the conditional mean and the conditional variance. While one may expect negative 'news' to have a stronger effect on volatility than positive ones, it may be interesting to see what the situation is when the unconditional mean is assumed to have some structure. For the asMA process in (3)

$$
\mu_{t}^{2}(\sigma)=\phi^{+2}\left(E \varepsilon_{t}^{+}\right)^{2}+\phi^{2} \varepsilon_{t-1}^{2}-2 \phi \phi^{+} \varepsilon_{t-1} E \varepsilon_{t}^{+}+\left(\phi^{+2}+2 \phi \phi^{+}\right) \varepsilon_{t-1}^{+2}-2 \phi^{+2} \varepsilon_{t-1}^{+} E \varepsilon_{t}^{+}
$$

In Figure 4 the top panels show the shock impact curves for a selection of parameters for the asMA process. The solid line represents the case in which the conditional mean only responds to negative shocks, whereas the other two curves represent models in which the effect of positive shocks is pronounced. Consider first the top right-hand panel, look at the dashed line $(\phi=0$, $\left.\phi^{+}= \pm 0.2\right)$ and compare it with the corresponding one in the top left-hand panel. Even if the conditional mean only responds to positive shocks, the impact of a shock can be larger for negative shocks than for positive ones as long as the persistence of the GARCH process is high. The bottom panels show the SIC when the conditional mean either follows an MA process or is constant. If the conditional mean is a linear MA process, the response to shocks due to the conditional mean is symmetric, $\mu_{t}^{2}(\sigma)=\phi^{2} \varepsilon_{t-1}^{2}$. In this case the effect of the conditional mean dominates the effect of the conditional variance so that the impact of a shock on the mean squared error is almost symmetric even if the GARCH process is asymmetric. A comparison of the solid lines in the bottom left and right-hand panels results in a similar conclusion in that the increased persistence in the GARCH process emphasizes the role of the conditional variance in the shock impact curve. Replacing the TGARCH process with a different asymmetric GARCH process has virtually no effect on the shape of the curves in Figure 4. A symmetric GARCH process would somewhat dampen the impact of negative shocks, in which case the curves in the bottom panels would be symmetric around zero.

For the GARCH-M process in (4),

$$
\mu_{t}^{2}(\sigma)=\phi^{2}\left(E h_{t}^{\delta}\right)^{2}+\phi^{2} h_{t}^{2 \delta}-2 \phi^{2} h_{t}^{\delta} E h_{t}^{\delta}
$$

In this case, the effect entering through the conditional variance is the one controlling the response to shocks. In Figure 5 the shock impact curves are plotted for TGARCH-M model with $\delta=1$ and 2 in (4). Comparing the left- and right-hand panels shows that increased persistence in the TGARCH process magnifies the impact of shocks. The asymmetry of the impact is inherited from the GARCH process. Replacing the TGARCH process with a symmetric GARCH process produces shock impact curves that are symmetric around zero.

\section{Conclusions}

In this paper we show how different parameterizations of the conditional mean and variance contribute to the asymmetry in the unconditional distribution of $y_{t}$. This is important because marginal distributions of return series often appear skewed. It is thus useful to know the structure 
Figure 4: asMA-TGARCH: Shock impact curves for different values for parameters for the conditional mean, TGARCH parameters: $\omega=0.005, \alpha=0.05, \beta=0.90$ (left-hand panels), $\beta=0.94$ (right-hand panels), and $\alpha^{*}=-0.04$.
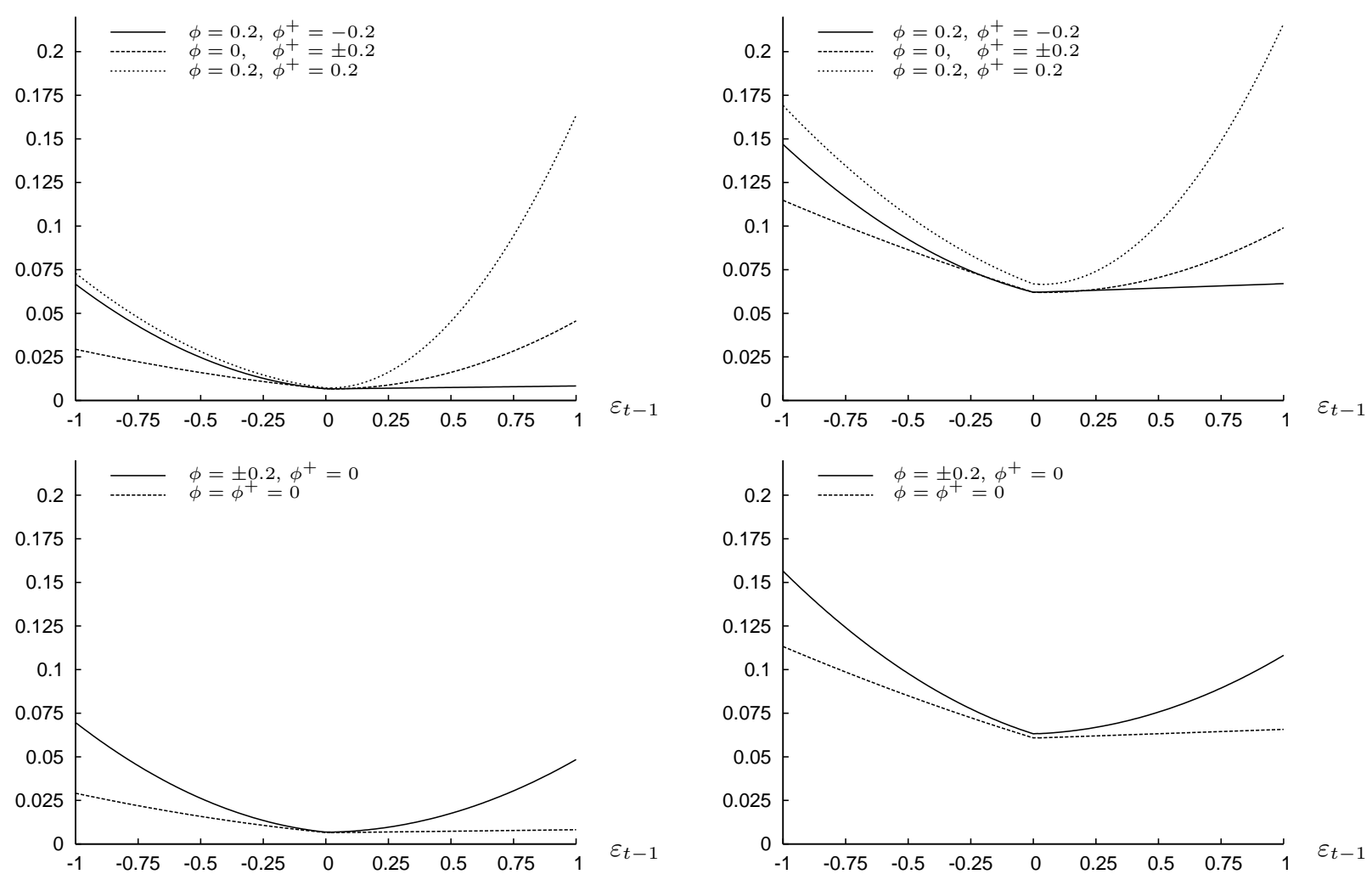

of the unconditional distribution implied by a model that has asymmetries or nonlinearities in the first and the second conditional moment.

The models we have considered in detail are the asMA-TGARCH and the TGARCH-M model. In the former model, both the conditional mean and the conditional standard deviation are asymmetric around zero. The latter model even has a nonlinear mean. We derive the analytic expressions for the third-order moment structure of these models and consider various special cases of the asMA-TGARCH model in which the mean and/or the standard deviation specification is restricted to be symmetric. Similar considerations are made in the case of the TGARCH-M model. In general, we find that asymmetries or nonlinearities in the conditional mean are of greater importance than they are in the conditional standard deviation or variance when it comes to generating skewed marginal distributions. If the conditional mean is symmetric and linear, then the unconditional skewness can only follow from the asymmetry of the conditional standard deviation or variance. However, in that case the third-moment structure of the variable of interest is no longer particularly flexible. But then, if the conditional mean is asymmetric or nonlinear, the distribution of $y_{t}$ can be even strongly skewed regardless of whether or not the conditional standard deviation or variance is symmetric or asymmetric.

It may be of interest to see how the past news affect not only the current volatility but magnitude of today's returns. We introduce a definition of the shock impact curve which describes the impact of a shock on the mean squared error of the return. It combines the effects of the conditional mean and the conditional standard deviation or variance on the squared returns. The conditional mean can strongly dominate the shape of the news impact curves. 
Figure 5: TGARCH-M: Shock impact curves for different values for parameters for the conditional mean, $\delta=1$ (top panels), $\delta=2$ (bottom panels); TGARCH parameters: $\omega=0.005, \alpha=0.05, \beta=0.90$ (left-hand panels), $\beta=0.94$ (right-hand panels), and $\alpha^{*}=-0.04$.
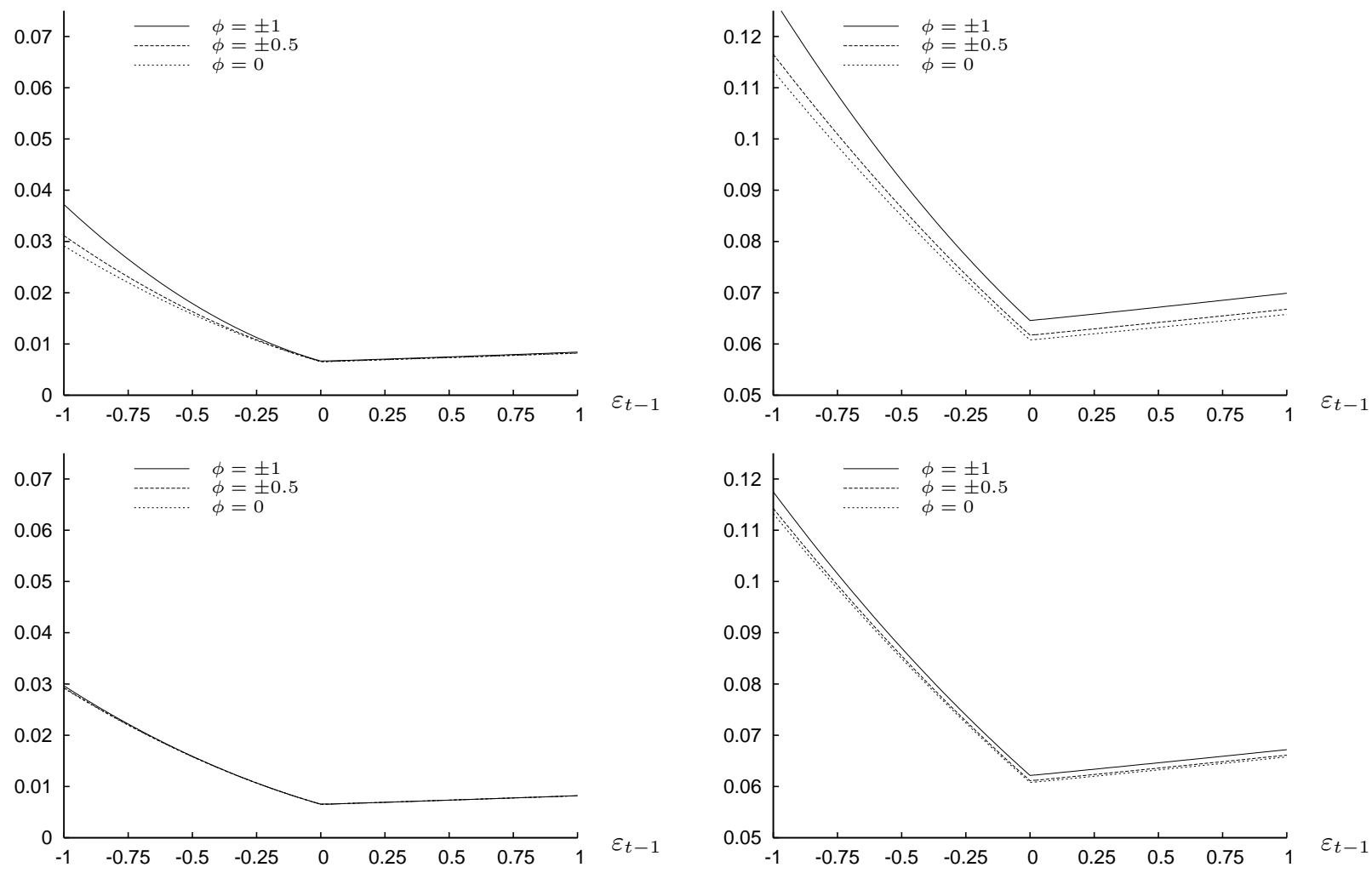

It would be interesting to consider a wider variety of specifications for the conditional mean and variance and derive the corresponding expressions in these cases. However, for many models, such as the standard GARCH model and some of its extensions, analytical expressions for thirdorder moments are not available. Our simulation experiments show that the same conclusions can be drawn when the TGARCH or AVGARCH process is replaced with other GARCH models that parameterize the conditional variance instead of the conditional standard deviation.

Finally, because a skewed marginal distribution can be a result of some type of asymmetric or nonlinear behaviour in the process for the conditional mean, testing for asymmetries and nonlinearities in the conditional mean is important. If an asymmetric or nonlinear model is found suitable, this may have implications on the unconditional third-moment structure of the process. Of course, any comparison of the unconditional moments estimated from the data with the moments implied by the fitted model (plug-in estimation) is dependent on simulations whenever analytical expressions for the moments of interest are not available. 


\section{References}

Bollerslev, T. (1986): "Generalized autoregressive conditional heteroskedasticity," Journal of Econometrics, 31, 307-327.

BrÄnnÄs, K., AND J. G. DE GoolJer (1995): "Invertibility of Nonlinear Time Series Models," Communications in Statistics: Theory and Methods, 24, 2701-2714.

(2004): "Asymmetries in conditional mean and variance: Modelling stock returns by asMA-asQGARCH," Journal of Forecasting, 23, 155-171.

BRÄNNÄs, K., AND N. NORDMAN (2003a): "An alternative conditional asymmetry specification for stock returns," Applied Financial Economics, 13, 537-541.

(2003b): "Conditional skewness modelling for stock returns," Applied Economic Letters, 10, 725-728.

Chen, J., H. Hong, And J. C. Stein (2001): "Forecasting crashes: trading volume, past returns, and conditional skewness in stock prices," Journal of Financial Economics, 61, 345381.

Engle, R. F. (1990): "Discussion: Stock market volatility and the crash of 87," Review of Financial Studies, 3, 103-106.

Engle, R. F., D. M. Lilien, and R. P. Robins (1987): "Estimating time varying risk premia in the term structure: the ARCH-M model," Econometrica, 55, 391-407.

ENGLE, R. F., AND V. K. NG (1993): "Measuring and testing the impact of news on volatility," Journal of Finance, 48, 1749-78.

Engle, R. F., And A. J. Patton (2001): "What good is a volatility model?," Quantitative Finance, 1, 237-245.

Glosten, L. W., R. Jagannathan, and D. E. Runkle (1993): "On the relation between the expected value and the volatility of the nominal excess return on stocks," Journal of Finance, 48, 1779-1801.

GonzÁlez-Rivera, G. (1998): "Smooth-Transition GARCH Models," Studies in Nonlinear Dynamics and Econometrics, 3, 61-78.

Hagerud, G. E. (1997): "Specification Tests for Asymmetric GARCH," Working Paper Series in Economics and Finance No. 163.

Hansen, B. E. (1994): "Autoregressive conditional density models," International Economic Review, 35, 705-730.

Harris, R., C. C. KüÇüKÖzmen, and F. Yilmaz (2004): "Skewness in the conditional distribution of daily equity returns," Applied Financial Economics, 14, 195-202.

Harvey, C. R., And A. Siddique (1999): "Autoregressive Conditional Skewness," The Journal of Financial and Quantitative Analysis, 34, 465-487.

He, C., And T. Teräsvirta (1999): "Fourth Moment Structure of the GARCH $(p, q)$ Process," Econometric Theory, 15, 824-846. 
Hentschel, L. (1995): "All in the family: Nesting symmetric and asymmetric GARCH models," Journal of Financial Economics, 39, 71-104.

Hong, E. P. (1991): "The autocorrelation structure for the GARCH-M process," Economics Letters, 37, 129-132.

Kim, T.-H., And H. White (2004): "On more robust estimation of skewness and kurtosis," Finance Research Letters, 1, 56-73.

Lambert, P., and S. Laurent (2002): "Modelling skewness dynamics in series of financial data using skewed location-scale distributions," Discussion Paper, Institut de Statistique, Louvain-la-Neuve.

Lanne, M., and P. Saikkonen (2005): "Modeling Conditional Skewness in Stock Returns," unpublished manuscript.

Ling, S., AND M. MCAleER (2002): "Stationarity and the existence of moments of a family of GARCH processes," Journal of Econometrics, 106, 109-117.

Pagan, A. R., And G. W. Schwert (1990): "Alternative models for conditional stock volatility," Journal of Econometrics, 45, 267-290.

Peiró, A. (2002): "Skewness in individual stocks at different investment horizons," Quantitative Finance, 2, 139-146.

(2004): "Asymmetries and tails in stock index returns: are their distributions really asymmetric?," Quantitative Finance, 4, 37-44.

SChWert, G. W. (1989): "Why does stock market volatility change over time?, Journal of Finance, 44, 1115-1153.

SentanA, E. (1995): "Quadratic ARCH Models," The Review of Economic Studies, 62, 639661.

TAylor, S. J. (1986): Modelling Financial Time Series. Chichester, UK: Wiley.

Wecker, W. E. (1981): "Asymmetric time series," Journal of the American Statistical Association, $76,16-21$.

ZaKoÏAn, J.-M. (1994): "Threshold heteroskedastic models," Journal of Economic Dynamics and Control, 18, 931-955. 


\section{Appendix}

Lemma 1 (First and second-moment structure of asMA) Consider an asymmetric MA process (1)-(3) and (5) that is second order stationary. The unconditional first and second-order moments and cross-moments of $y_{t}$ are given by

$$
\begin{aligned}
E y_{t} & =0 \\
\operatorname{Var} y_{t} & =E \mu_{t}^{2}+E \varepsilon_{t}^{2}, \\
\gamma_{11}(k) & =E \mu_{t} \mu_{t-k}+E \mu_{t} \varepsilon_{t-k}, \quad k \geq 1,
\end{aligned}
$$

where

$$
\begin{aligned}
E \mu_{t}^{2} & =\phi^{2} E \varepsilon_{t}^{2}+\left(2 \phi \phi^{+}+\phi^{+2}\right) E \varepsilon_{t}^{+2}-\phi^{+2}\left(E \varepsilon_{t}^{+}\right)^{2} \\
E \mu_{t} \mu_{t-k} & =\phi \phi^{+} E \varepsilon_{t-k} \varepsilon_{t}^{+}+\phi^{+2} E \varepsilon_{t}^{+} \varepsilon_{t-k}^{+}-\phi^{+2}\left(E \varepsilon_{t}^{+}\right)^{2} \\
E \mu_{t} \varepsilon_{t-k} & = \begin{cases}\phi E \varepsilon_{t}^{2}+\phi^{+} E \varepsilon_{t}^{+2}, & k=1 \\
\phi^{+} E \varepsilon_{t-k} \varepsilon_{t-1}^{+}, & k>1 .\end{cases}
\end{aligned}
$$

The moments of $\varepsilon_{t}$ and $\varepsilon_{t}^{+}$can be found in Lemma 5 .

Proof. The results are readily obtained by straightforward algebra.

Lemma 2 (Third-moment structure of asMA) Consider an asymmetric MA process (1)(3) and (5) that is third-order stationary. The unconditional third-order moments and crossmoments of $y_{t}$ are given by

$$
\begin{aligned}
\gamma_{3} & =E \mu_{t}^{3}+3 E \mu_{t} \varepsilon_{t}^{2}, & \\
\gamma_{21}(k) & =E \mu_{t}^{2} \mu_{t-k}+E \mu_{t-k} \varepsilon_{t}^{2}+E \mu_{t}^{2} \varepsilon_{t-k}+E \varepsilon_{t}^{2} \varepsilon_{t-k}, & k \geq 1 \\
\gamma_{12}(k) & =E \mu_{t} \mu_{t-k}^{2}+E \mu_{t} \varepsilon_{t-k}^{2}+2 E \mu_{t} \mu_{t-k} \varepsilon_{t-k}, & k \geq 1
\end{aligned}
$$

where

$$
\begin{aligned}
E \mu_{t}^{3}= & \left(3 \phi^{2} \phi^{+}+3 \phi \phi^{+2}+\phi^{+3}\right) E \varepsilon_{t}^{+3}-\left(6 \phi \phi^{+2}+3 \phi^{+3}\right) E \varepsilon_{t}^{+2} E \varepsilon_{t}^{+}-3 \phi^{2} \phi^{+} E \varepsilon_{t}^{2} E \varepsilon_{t}^{+} \\
& +2 \phi^{+3}\left(E \varepsilon_{t}^{+}\right)^{3} \\
E \mu_{t}^{2} \mu_{t-k}= & \phi^{3} E \varepsilon_{t}^{2} \varepsilon_{t-k}+\left(2 \phi^{2} \phi^{+}+\phi \phi^{+2}\right) E \varepsilon_{t-k} \varepsilon_{t}^{+2} \\
& +\phi^{2} \phi^{+}\left(E \varepsilon_{t}^{2} \varepsilon_{t-k}^{+}-E \varepsilon_{t}^{2} E \varepsilon_{t}^{+}\right)+\left(2 \phi \phi^{+2}+\phi^{+3}\right)\left(E \varepsilon_{t}^{+2} \varepsilon_{t-k}^{+}-E \varepsilon_{t}^{+2} E \varepsilon_{t}^{+}\right) \\
& -2 \phi \phi^{+2} E \varepsilon_{t-k} \varepsilon_{t}^{+} E \varepsilon_{t}^{+}-2 \phi^{+3}\left(E \varepsilon_{t}^{+} \varepsilon_{t-k}^{+} E \varepsilon_{t}^{+}-\left(E \varepsilon_{t}^{+}\right)^{3}\right) \\
E \mu_{t} \mu_{t-k}^{2}= & \phi^{2} \phi^{+}\left(E \varepsilon_{t-k}^{2} \varepsilon_{t}^{+}-E \varepsilon_{t}^{2} E \varepsilon_{t}^{+}\right)+\left(2 \phi \phi^{+2}+\phi^{+3}\right)\left(E \varepsilon_{t}^{+} \varepsilon_{t-k}^{+2}-E \varepsilon_{t}^{+2} E \varepsilon_{t}^{+}\right) \\
& -2 \phi \phi^{+2} E \varepsilon_{t-k} \varepsilon_{t}^{+} E \varepsilon_{t}^{+}-2 \phi^{+3}\left(E \varepsilon_{t}^{+} \varepsilon_{t-k}^{+} E \varepsilon_{t}^{+}-\left(E \varepsilon_{t}^{+}\right)^{3}\right) \\
E \mu_{t} \varepsilon_{t}^{2}= & \phi E \varepsilon_{t}^{2} \varepsilon_{t-1}+\phi^{+}\left(E \varepsilon_{t}^{2} \varepsilon_{t-1}^{+}-E \varepsilon_{t}^{2} E \varepsilon_{t}^{+}\right) \\
E \mu_{t-k} \varepsilon_{t}^{2}= & \phi E \varepsilon_{t}^{2} \varepsilon_{t-k-1}+\phi^{+}\left(E \varepsilon_{t}^{2} \varepsilon_{t-k-1}^{+}-E \varepsilon_{t}^{2} E \varepsilon_{t}^{+}\right)
\end{aligned}
$$




$$
\begin{aligned}
& E \mu_{t} \varepsilon_{t-k}^{2}= \begin{cases}\phi^{+}\left(E \varepsilon_{t}^{+3}-E \varepsilon_{t}^{2} E \varepsilon_{t}^{+}\right), & k=1 \\
\phi^{+}\left(E \varepsilon_{t-(k-1)}^{2} \varepsilon_{t}^{+}-E \varepsilon_{t}^{2} E \varepsilon_{t}^{+}\right), & k>1\end{cases} \\
& E \mu_{t}^{2} \varepsilon_{t-k}= \begin{cases}\left(2 \phi \phi^{+}+\phi^{+2}\right) E \varepsilon_{t}^{+3}-2 \phi \phi^{+} E \varepsilon_{t}^{2} E \varepsilon_{t}^{+}-2 \phi^{+2} E \varepsilon_{t}^{+2} E \varepsilon_{t}^{+}, \quad k=1 \\
\left(2 \phi \phi^{+}+\phi^{+2}\right) E \varepsilon_{t-(k-1)} \varepsilon_{t}^{+2}-2 \phi^{+2} E \varepsilon_{t-(k-1)} \varepsilon_{t}^{+} E \varepsilon_{t}^{+} \\
+\phi^{2} E \varepsilon_{t}^{2} \varepsilon_{t-(k-1)},\end{cases} \\
& E \mu_{t} \mu_{t-k} \varepsilon_{t-k}= \begin{cases}\phi \phi^{+}\left(E \varepsilon_{t}^{2} \varepsilon_{t-1}^{+}-E \varepsilon_{t}^{2} E \varepsilon_{t}^{+}+E \varepsilon_{t-1} \varepsilon_{t}^{+2}\right)+\phi^{+2}\left(E \varepsilon_{t}^{+2} \varepsilon_{t-1}^{+}-E \varepsilon_{t}^{+2} E \varepsilon_{t}^{+}\right) \\
+\phi^{2} E \varepsilon_{t}^{2} \varepsilon_{t-1}, \\
\phi \phi^{+} E \varepsilon_{t-(k-1)} \varepsilon_{t-k} \varepsilon_{t}^{+}+\phi^{+2}\left(E \varepsilon_{t-(k-1)} \varepsilon_{t}^{+} \varepsilon_{t-k}^{+}-E \varepsilon_{t-(k-1)} \varepsilon_{t}^{+} E \varepsilon_{t}^{+}\right), & k>1 .\end{cases}
\end{aligned}
$$

The moments of $\varepsilon_{t}$ and $\varepsilon_{t}^{+}$can be found in Lemma 5.

Proof. The results are readily obtained by tedious but straightforward algebra.

Corollary 1 (Third-moment structure of asMA with symmetric GARCH) Consider an asymmetric MA process (1)-(3) and (5) that is third-order stationary. Furthermore, assume that $c_{t}$ in (5) is even with respect to $z_{t}$. The unconditional third-order moments and cross-moments of $y_{t}$ are given by (11)-(13) in Lemma 2 where

$$
\begin{aligned}
& E \mu_{t}^{3}=\left(3 \phi^{2} \phi^{+}+3 \phi \phi^{+2}+\phi^{+3}\right) E \varepsilon_{t}^{+3}-\left(6 \phi \phi^{+2}+3 \phi^{+3}\right) E \varepsilon_{t}^{+2} E \varepsilon_{t}^{+} \\
& -3 \phi^{2} \phi^{+} E \varepsilon_{t}^{2} E \varepsilon_{t}^{+}+2 \phi^{+3}\left(E \varepsilon_{t}^{+}\right)^{3} \\
& E \mu_{t}^{2} \mu_{t-k}=\phi^{2} \phi^{+}\left(E \varepsilon_{t}^{2} \varepsilon_{t-k}^{+}-E \varepsilon_{t}^{2} E \varepsilon_{t}^{+}\right)+\left(2 \phi \phi^{+2}+\phi^{+3}\right)\left(E \varepsilon_{t}^{+2} \varepsilon_{t-k}^{+}-E \varepsilon_{t}^{+2} E \varepsilon_{t}^{+}\right) \\
& -2 \phi^{+3}\left(E \varepsilon_{t}^{+} \varepsilon_{t-k}^{+} E \varepsilon_{t}^{+}-\left(E \varepsilon_{t}^{+}\right)^{3}\right) \\
& E \mu_{t} \mu_{t-k}^{2}=\phi^{2} \phi^{+}\left(E \varepsilon_{t-k}^{2} \varepsilon_{t}^{+}-E \varepsilon_{t}^{2} E \varepsilon_{t}^{+}\right)+\left(2 \phi \phi^{+2}+\phi^{+3}\right)\left(E \varepsilon_{t}^{+} \varepsilon_{t-k}^{+2}-E \varepsilon_{t}^{+2} E \varepsilon_{t}^{+}\right) \\
& -2 \phi^{+3}\left(E \varepsilon_{t}^{+} \varepsilon_{t-k}^{+} E \varepsilon_{t}^{+}-\left(E \varepsilon_{t}^{+}\right)^{3}\right) \\
& E \mu_{t} \varepsilon_{t}^{2}=\phi^{+}\left(E \varepsilon_{t}^{2} \varepsilon_{t-1}^{+}-E \varepsilon_{t}^{2} E \varepsilon_{t}^{+}\right) \\
& E \mu_{t-k} \varepsilon_{t}^{2}=\phi^{+}\left(E \varepsilon_{t}^{2} \varepsilon_{t-k-1}^{+}-E \varepsilon_{t}^{2} E \varepsilon_{t}^{+}\right) \\
& E \mu_{t} \varepsilon_{t-k}^{2}= \begin{cases}\phi^{+}\left(E \varepsilon_{t}^{+3}-E \varepsilon_{t}^{2} E \varepsilon_{t}^{+}\right), & k=1 \\
\phi^{+}\left(E \varepsilon_{t-k}^{2} \varepsilon_{t-1}^{+}-E \varepsilon_{t}^{2} E \varepsilon_{t}^{+}\right), & k>1\end{cases} \\
& E \mu_{t}^{2} \varepsilon_{t-k}= \begin{cases}\left(2 \phi \phi^{+}+\phi^{+2}\right) E \varepsilon_{t}^{+3}-2 \phi_{1} \phi_{1}^{+} E \varepsilon_{t}^{2} E \varepsilon_{t}^{+}-2 \phi_{1}^{+2} E \varepsilon_{t}^{+2} E \varepsilon_{t}^{+}, & k=1 \\
0, & k>1\end{cases} \\
& E \mu_{t} \mu_{t-k} \varepsilon_{t-k}= \begin{cases}\phi \phi^{+}\left(E \varepsilon_{t}^{2} \varepsilon_{t-1}^{+}-E \varepsilon_{t}^{2} E \varepsilon_{t}^{+}+E \varepsilon_{t-1} \varepsilon_{t}^{+2}\right)+\phi^{+2}\left(E \varepsilon_{t}^{+2} \varepsilon_{t-1}^{+}-E \varepsilon_{t}^{+2} E \varepsilon_{t}^{+}\right), & k=1 \\
0, & k>1 .\end{cases}
\end{aligned}
$$

The moments of $\varepsilon_{t}$ and $\varepsilon_{t}^{+}$can be found in Lemma 5 . 
Corollary 2 (Third-moment structure of asMA with $\phi=0$ ) Consider an asymmetric MA process (1)-(3) and (5) with $\phi=0$ that is third-order stationary. The unconditional third-order moments and cross-moments of $y_{t}$ are given by (11)-(13) in Lemma 2 where

$$
\begin{aligned}
& E \mu_{t}^{3}=\phi^{+3}\left(E \varepsilon_{t}^{+3}-3 E \varepsilon_{t}^{+2} E \varepsilon_{t}^{+}+2\left(E \varepsilon_{t}^{+}\right)^{3}\right) \\
& E \mu_{t}^{2} \mu_{t-k}=\phi^{+3}\left(E \varepsilon_{t}^{+2} \varepsilon_{t-k}^{+}-E \varepsilon_{t}^{+2} E \varepsilon_{t}^{+}\right)-2 \phi^{+3}\left(E \varepsilon_{t}^{+} \varepsilon_{t-k}^{+} E \varepsilon_{t}^{+}-\left(E \varepsilon_{t}^{+}\right)^{3}\right) \\
& E \mu_{t} \mu_{t-k}^{2}=\phi^{+3}\left(E \varepsilon_{t}^{+} \varepsilon_{t-k}^{+2}-E \varepsilon_{t}^{+2} E \varepsilon_{t}^{+}\right)-2 \phi^{+3}\left(E \varepsilon_{t}^{+} \varepsilon_{t-k}^{+} E \varepsilon_{t}^{+}-\left(E \varepsilon_{t}^{+}\right)^{3}\right) \\
& E \mu_{t} \varepsilon_{t}^{2}=\phi^{+}\left(E \varepsilon_{t}^{2} \varepsilon_{t-1}^{+}-E \varepsilon_{t}^{2} E \varepsilon_{t}^{+}\right) \\
& E \mu_{t-k} \varepsilon_{t}^{2}=\phi^{+}\left(E \varepsilon_{t}^{2} \varepsilon_{t-k-1}^{+}-E \varepsilon_{t}^{2} E \varepsilon_{t}^{+}\right) \\
& E \mu_{t} \varepsilon_{t-k}^{2}= \begin{cases}\phi^{+}\left(E \varepsilon_{t}^{+3}-E \varepsilon_{t}^{2} E \varepsilon_{t}^{+}\right), & k=1 \\
\phi^{+}\left(E \varepsilon_{t-k}^{2} \varepsilon_{t-1}^{+}-E \varepsilon_{t}^{2} E \varepsilon_{t}^{+}\right), & k>1\end{cases} \\
& E \mu_{t}^{2} \varepsilon_{t-k}= \begin{cases}\phi^{+2}\left(E \varepsilon_{t}^{+3}-2 E \varepsilon_{t}^{+2} E \varepsilon_{t}^{+}\right), & k=1 \\
\phi^{+2}\left(E \varepsilon_{t-k} \varepsilon_{t-1}^{+2}-2 E \varepsilon_{t-k} \varepsilon_{t-1}^{+} E \varepsilon_{t}^{+}\right), & k>1\end{cases} \\
& E \mu_{t} \mu_{t-k} \varepsilon_{t-k}= \begin{cases}\phi^{+2}\left(E \varepsilon_{t}^{+2} \varepsilon_{t-1}^{+}-E \varepsilon_{t}^{+2} E \varepsilon_{t}^{+}\right), & k=1 \\
\phi^{+2}\left(E \varepsilon_{t-k} \varepsilon_{t-1}^{+} \varepsilon_{t-k-1}^{+}-E \varepsilon_{t-k} \varepsilon_{t-1}^{+} E \varepsilon_{t}^{+}\right), & k>1 .\end{cases}
\end{aligned}
$$

The moments of $\varepsilon_{t}$ and $\varepsilon_{t}^{+}$can be found in Lemma 5 .

Corollary 3 (Third-moment structure of asMA with $\phi=0$ and symmetric GARCH) Consider an asymmetric MA process (1)-(3) and (5) with $\phi=0$ that is third-order stationary. Furthermore, assume that $c_{t}$ in (5) is even with respect to $z_{t}$. The unconditional third-order moments and cross-moments of $y_{t}$ are given by (11)-(13) in Lemma 2 where

$$
\begin{aligned}
E \mu_{t}^{3} & =\phi^{+3}\left(E \varepsilon_{t}^{+3}-3 E \varepsilon_{t}^{+2} E \varepsilon_{t}^{+}+2\left(E \varepsilon_{t}^{+}\right)^{3}\right) \\
E \mu_{t}^{2} \mu_{t-k} & =\phi^{+3} E \varepsilon_{t}^{+2} \varepsilon_{t-k}^{+}-\phi^{+3} E \varepsilon_{t}^{+2} E \varepsilon_{t}^{+}-2 \phi^{+3}\left(E \varepsilon_{t}^{+} \varepsilon_{t-k}^{+} E \varepsilon_{t}^{+}-\left(E \varepsilon_{t}^{+}\right)^{3}\right) \\
E \mu_{t} \mu_{t-k}^{2} & =\phi^{+3} E \varepsilon_{t}^{+} \varepsilon_{t-k}^{+2}-\phi^{+3} E \varepsilon_{t}^{+2} E \varepsilon_{t}^{+}-2 \phi^{+3}\left(E \varepsilon_{t}^{+} \varepsilon_{t-k}^{+} E \varepsilon_{t}^{+}-\left(E \varepsilon_{t}^{+}\right)^{3}\right) \\
E \mu_{t} \varepsilon_{t}^{2} & =\phi^{+}\left(E \varepsilon_{t}^{2} \varepsilon_{t-1}^{+}-E \varepsilon_{t}^{2} E \varepsilon_{t}^{+}\right) \\
E \mu_{t-k} \varepsilon_{t}^{2} & =\phi^{+}\left(E \varepsilon_{t}^{2} \varepsilon_{t-k-1}^{+}-E \varepsilon_{t}^{2} E \varepsilon_{t}^{+}\right) \\
E \mu_{t} \varepsilon_{t-k}^{2} & = \begin{cases}\phi^{+}\left(E \varepsilon_{t}^{+3}-E \varepsilon_{t}^{2} E \varepsilon_{t}^{+}\right), & k=1 \\
\phi^{+}\left(E \varepsilon_{t-k}^{2} \varepsilon_{t-1}^{+}-E \varepsilon_{t}^{2} E \varepsilon_{t}^{+}\right), & k>1\end{cases} \\
E \mu_{t}^{2} \varepsilon_{t-k} & = \begin{cases}\phi^{+2}\left(E \varepsilon_{t}^{+3}-2 E \varepsilon_{t}^{+2} E \varepsilon_{t}^{+}\right), & k=1 \\
0, & k>1\end{cases} \\
E \mu_{t} \mu_{t-k} \varepsilon_{t-k} & = \begin{cases}\phi^{+2}\left(E \varepsilon_{t}^{+2} \varepsilon_{t-1}^{+}-E \varepsilon_{t}^{+2} E \varepsilon_{t}^{+}\right), & k=1 \\
0, & k>1 .\end{cases}
\end{aligned}
$$

The moments of $\varepsilon_{t}$ and $\varepsilon_{t}^{+}$can be found in Lemma 5. 
Corollary 4 (Third-moment structure of MA) Consider an asymmetric MA process (1)(3) and (5) with $\phi^{+}=0$ that is third-order stationary. The unconditional third-order moments and cross-moments of $y_{t}$ are given by (11)-(13) in Lemma 2 where

$$
E \mu_{t}^{3}=E \mu_{t} \mu_{t-k}^{2}=E \mu_{t} \varepsilon_{t-k}^{2}=0
$$

and

$$
\begin{aligned}
E \mu_{t} \varepsilon_{t}^{2} & =\phi E \varepsilon_{t}^{2} \varepsilon_{t-1} \\
E \mu_{t}^{2} \mu_{t-k} & =\phi^{3} E \varepsilon_{t}^{2} \varepsilon_{t-k} \\
E \mu_{t-k} \varepsilon_{t}^{2} & =\phi E \varepsilon_{t}^{2} \varepsilon_{t-(k+1)} \\
E \mu_{t}^{2} \varepsilon_{t-k} & = \begin{cases}0, & k=1 \\
\phi^{2} E \varepsilon_{t}^{2} \varepsilon_{t-(k-1)}, & k>1\end{cases} \\
E \mu_{t} \mu_{t-k} \varepsilon_{t-k} & = \begin{cases}\phi^{2} E \varepsilon_{t}^{2} \varepsilon_{t-1}, & k=1 \\
0, & k>1 .\end{cases}
\end{aligned}
$$

The moments of $\varepsilon_{t}$ and $\varepsilon_{t}^{+}$can be found in Lemma 5 .

If the conditional second moment is parameterized such that $E \varepsilon_{t} \varepsilon_{t-k}=0$ for all $k \geq 1$ (for instance $c_{t}$ in (5) is symmetric with respect to $z_{t}$ ), then the third-order moments and crossmoments are zero.

Lemma 3 (First and second-moment structure of GARCH-M) Consider a $G A R C H-M$ process (1), (2), (4), and (5) that is third-order stationary. The unconditional first and secondorder moments and cross-moments of $y_{t}$ are given by (8)-(10) in Lemma 1 where

$$
\begin{aligned}
E \mu_{t}^{2} & =\phi^{2}\left(E h_{t}^{2 \delta}-\left(E h_{t}^{\delta}\right)^{2}\right) \\
E \mu_{t} \mu_{t-k} & =\phi^{2}\left(E h_{t}^{\delta} h_{t-k}^{\delta}-\left(E h_{t}^{\delta}\right)^{2}\right) \\
E \mu_{t} \varepsilon_{t-k} & =\phi E h_{t}^{\delta} \varepsilon_{t-k} .
\end{aligned}
$$

The moments of $\varepsilon_{t}$ and $h_{t}$ can be found in Lemma 5.

Proof. The results are readily obtained by straightforward algebra.

Lemma 4 (Third-moment structure of GARCH-M) Consider a GARCH-M process (1), (2), (4), and (5) that is third-order stationary. The unconditional third-order moments and cross-moments of $y_{t}$ are given by (11)-(13) in Lemma 2 where

$$
\begin{aligned}
E \mu_{t}^{3} & =\phi^{3}\left(E h_{t}^{3 \delta}-3 E h_{t}^{2 \delta} E h_{t}^{\delta}+2\left(E h_{t}^{\delta}\right)^{3}\right) \\
E \mu_{t}^{2} \mu_{t-k} & =\phi^{3}\left(E h_{t}^{2 \delta} h_{t-k}^{\delta}-2 E h_{t}^{\delta} h_{t-k}^{\delta} E h_{t}^{\delta}-E h_{t}^{2 \delta} E h_{t}^{\delta}+2\left(E h_{t}^{\delta}\right)^{3}\right) \\
E \mu_{t} \mu_{t-k}^{2} & =\phi^{3}\left(E h_{t}^{\delta} h_{t-k}^{2 \delta}-2 E h_{t}^{\delta} h_{t-k}^{\delta} E h_{t}^{\delta}-E h_{t}^{2 \delta} E h_{t}^{\delta}+2\left(E h_{t}^{\delta}\right)^{3}\right)
\end{aligned}
$$




$$
\begin{aligned}
E \mu_{t}^{2} \varepsilon_{t-k} & =\phi^{2}\left(E h_{t}^{2 \delta} \varepsilon_{t-k}-2 E h_{t}^{\delta} \varepsilon_{t-k} E h_{t}^{\delta}\right) \\
E \mu_{t} \mu_{t-k} \varepsilon_{t-k} & =\phi^{2}\left(E h_{t}^{\delta} h_{t-k}^{\delta} \varepsilon_{t-k}-E h_{t}^{\delta} \varepsilon_{t-k} E h_{t}^{\delta}\right) \\
E \mu_{t} \varepsilon_{t}^{2} & =\phi\left(E h_{t}^{\delta+2}-E h_{t}^{\delta} E h_{t}^{2}\right) \\
E \mu_{t} \varepsilon_{t-k}^{2} & =\phi\left(E h_{t}^{\delta} \varepsilon_{t-k}^{2}-E h_{t}^{\delta} E h_{t}^{2}\right) \\
E \mu_{t-k} \varepsilon_{t}^{2} & =\phi\left(E h_{t}^{2} h_{t-k}^{\delta}-E h_{t}^{\delta} E h_{t}^{2}\right) .
\end{aligned}
$$

The moments of $\varepsilon_{t}$ and $\varepsilon_{t}^{+}$can be found in Lemma 5. If the $c_{t}$ in (5) is even with respect to $z_{t}$, then $E \mu_{t}^{2} \varepsilon_{t-k}=E \mu_{t} \mu_{t-k} \varepsilon_{t-k}=0$.

Proof. The results are readily obtained by straightforward algebra. For the results for the symmetric GARCH we make use of Corollary 5.

Lemma 5 Consider the GARCH model (2) and (5). Suppose that $\varepsilon_{t}$ is stationary with timeinvariant moments. Provided that the moments exist, the moments of $\varepsilon_{t}$, $\varepsilon_{t}^{+}$, and $h_{t}$ are given by

$$
\begin{aligned}
E \varepsilon_{t}^{m} & = \begin{cases}d_{0 m} E h_{t}^{m}, & \text { m even } \\
0, & \text { m odd }\end{cases} \\
E \varepsilon_{t}^{+m} & =d_{0 m}^{+} E h_{t}^{m} \\
E \varepsilon_{t}^{m} \varepsilon_{t-k}^{(+) n} & = \begin{cases}d_{0 m} E h_{t}^{m} \varepsilon_{t-k}^{(+)}, & \text {m even } \\
0, & m \text { odd }\end{cases} \\
E \varepsilon_{t}^{+m} \varepsilon_{t-k}^{(+) n} & =d_{0 m}^{+} E h_{t}^{m} \varepsilon_{t-k}^{(+) n} \\
E \varepsilon_{t-k} \varepsilon_{t}^{+} \varepsilon_{t-k-1}^{(+)} & =d_{01}^{+} E h_{t} \varepsilon_{t-k} \varepsilon_{t-k-1}^{(+)}
\end{aligned}
$$

where

$$
\begin{aligned}
E h_{t}^{d m} & =\frac{1}{1-d_{m 0}} \sum_{j=1}^{m}\left(\begin{array}{c}
m \\
j
\end{array}\right) \omega^{j} d_{(m-j) 0} E h_{t}^{d(m-j)} \\
E h_{t}^{d n} \varepsilon_{t-k}^{(+) m} & = \begin{cases}\sum_{j=0}^{n}\left(\begin{array}{c}
n \\
j
\end{array}\right) \omega^{j} d_{(n-j) m}^{(+)} E h_{t}^{d(n-j)+m}, & k=1 \\
\sum_{j=0}^{n}\left(\begin{array}{c}
n \\
j
\end{array}\right) \omega^{j} d_{(n-j) 0} E h_{t}^{d(n-j)} \varepsilon_{t-(k-1)}^{(+) m}, & k>1\end{cases} \\
E h_{t}^{d} \varepsilon_{t-k} \varepsilon_{t-k-1}^{(+)} & =d_{10}^{k-1} d_{11} E h_{t}^{d+1} \varepsilon_{t-1}^{(+)}
\end{aligned}
$$

and

$$
\begin{aligned}
E h_{t}^{d n} h_{t-k}^{d m} & = \begin{cases}\sum_{j=0}^{n}\left(\begin{array}{c}
n \\
j
\end{array}\right) \omega^{j} d_{(n-j) 0} E h_{t}^{d(n-j+m)}, & k=1 \\
\sum_{j=0}^{n}\left(\begin{array}{c}
n \\
j
\end{array}\right) \omega^{j} d_{(n-j) 0} E h_{t}^{d(n-j)} h_{t-(k-1)}^{d m}, & k>1\end{cases} \\
E h_{t}^{d} h_{t-k}^{d} \varepsilon_{t-k} & =d_{10}^{k-1} d_{11} E h_{t}^{2 d+1}
\end{aligned}
$$

with $k \geq 1$ and where

$$
\begin{aligned}
d_{i j}=E c_{t}^{i} z_{t}^{j}, & i \geq 0, \quad j \geq 0 \\
d_{i j}^{+}=E c_{t}^{i} z_{t}^{+j}, & i \geq 0, \quad j>0
\end{aligned}
$$


In the expressions above the notation $(+)$ means that + is either included in or excluded from the equation in question. When $d=1$ the recursions above yield analytically explicit expressions whereas for $d=2$ some of them involve moments that have to be calculated numerically through simulations. If the conditional standard deviation follows the TGARCH process (5) and (6) then

$$
\begin{aligned}
d_{i j} & =\sum_{\substack{0 \leq h_{1}, h_{2}, h_{3} \leq i \\
h_{1}+h_{2}+h_{3}=i \\
h_{3}+j \text { even }}} \frac{i !}{h_{1} ! h_{2} ! h_{3} !} \alpha^{h_{1}} \beta^{h_{2}} \alpha^{* h_{3}} E\left|z_{t}\right|^{i+j-h_{2}}, \quad i \geq 1, j \geq 0 \\
d_{i j}^{+} & =\sum_{\substack{0 \leq h_{1}, h_{2}, h_{3} \leq i \\
h_{1}+h_{2}+h_{3}=i}} \frac{i !}{h_{1} ! h_{2} ! h_{3} !} \alpha^{h_{1}} \beta^{h_{2}} \alpha^{* h_{3}} E z_{t}^{+i+j-h_{2}}, \quad i \geq 1, j \geq 1
\end{aligned}
$$

For the AVGARCH process the expressions for $d_{i j}$ and $d_{i j}^{+}$are obtained by setting $\alpha^{*}=0$, restricting the index $h_{3}=0$, and defining $0^{0}=1$. Furthermore, if $z_{t} \sim \operatorname{nid}(0,1)$, then the moments of $z_{t},\left|z_{t}\right|$, and the censored variable $z_{t}^{+}$are given in Lemma 6.

Proof. The results are readily obtained by straightforward but tedious algebra.

Corollary 5 Consider the GARCH model (2) and (5) in Lemma 5. If the process for the conditional second moment is symmetric in its response to shocks, then $d_{n m}=0$ whenever $m$ is odd. Hence, of the moments in Lemma 5,

$$
E h_{t}^{d n} \varepsilon_{t-k}^{m}=E h_{t}^{d} h_{t-k}^{d} \varepsilon_{t-k}=E h_{t}^{d} \varepsilon_{t-k} \varepsilon_{t-k-1}^{(+)}=0
$$

and

$$
E \varepsilon_{t}^{n} \varepsilon_{t-k}^{m}=E \varepsilon_{t}^{+d n} \varepsilon_{t-k}^{m}=E \varepsilon_{t-k} \varepsilon_{t}^{+} \varepsilon_{t-k-1}^{(+)}=0
$$

where $m$ is odd.

Proof. If $c_{t}\left(z_{t}\right)$ in (5) is an even function of $z_{t}$ then the function $c_{t}^{n}\left(z_{t}\right) z_{t}^{m}$ is an odd function of $z_{t}$ for any odd $m$ and therefore $d_{n m}=0$.

Lemma 6 Assume $z_{t} \sim \operatorname{nid}(0,1)$. Then the moments of $z_{t},\left|z_{t}\right|$, and $z_{t}^{+}$are given by

$$
\begin{aligned}
E z_{t}^{m} & = \begin{cases}\prod_{i=1}^{m-1} i, & m \text { even } \\
i \text { odd } & \\
0, & m \text { odd }\end{cases} \\
E\left|z_{t}\right|^{m} & = \begin{cases}E z_{t}^{m}, & m \text { even } \\
\prod_{i=2}^{m-1} i, & m \text { odd } \\
i \text { even }\end{cases} \\
E z_{t}^{+m} & =\frac{1}{2} E\left|z_{t}\right|^{m} .
\end{aligned}
$$

Proof. Straightforward by recursion and by noticing that the censored variable $z_{t}^{+}$can be expressed as

$$
z_{t}^{+}=\max \left(0, z_{t}\right)=\frac{1}{2}\left(\left|z_{t}\right|+z_{t}\right)
$$

Note that an empty product is defined to equal one. 